This item was submitted to Loughborough's Research Repository by the author.

Items in Figshare are protected by copyright, with all rights reserved, unless otherwise indicated.

\title{
Adaptive output regulation for a class of nonlinear systems with guaranteed transient performance
}

PLEASE CITE THE PUBLISHED VERSION

https://doi.org/10.1177/0142331219886411

PUBLISHER

SAGE Publications

VERSION

AM (Accepted Manuscript)

\section{PUBLISHER STATEMENT}

This paper was accepted for publication in the journal Transactions of the Institute of Measurement and Control and the definitive published version is available at https://doi.org/10.1177/0142331219886411. Users who receive access to an article through a repository are reminded that the article is protected by copyright and reuse is restricted to non-commercial and no derivative uses. Users may also download and save a local copy of an article accessed in an institutional repository for the user's personal reference.

\section{LICENCE}

CC BY-NC-ND 4.0

\section{REPOSITORY RECORD}

Sun, Weijie, Zhenhua Zhu, Jianglin Lan, and Yunjian Peng. 2019. "Adaptive Output Regulation for a Class of Nonlinear Systems with Guaranteed Transient Performance”. Loughborough University. https://hdl.handle.net/2134/12048585.v1. 


\title{
Adaptive Output
}

\section{Regulation for a Class}

of Nonlinear Systems

with Guaranteed

\section{Transient Performance}

Journal Title

$X X(X): 2-30$

(C)The Author(s) 2016

Reprints and permission:

sagepub.co.uk/journalsPermissions.nav

DOI: $10.1177 /$ ToBeAssigned

www.sagepub.com/

SAGE

\section{Weijie Sun ${ }^{1}$, Zhenhua Zhu' ${ }^{1}$, Jianglin Lan ${ }^{2}$ and Yunjian Peng ${ }^{1}$}

\begin{abstract}
This paper is dedicated to adaptive output regulation for a class of nonlinear systems with asymptotic output tracking and guarantee of prescribed transient performance. With the employment of internal model principle, we first transform this problem into a specific adaptive stabilization problem with output constraints. Then, by integrating the time-varying Barrier Lyapunov Function (BLF) technique together with the high gain feedback method, we develop an output-based control law to solve the constrained stabilization problem and consequently confine the output tracking error to a predefined arbitrary region. The output-based control law enables adaptive output regulation in the sense that, under unknown exosystem dynamics, all the closed-loop system signals are bounded whilst the controlled output constraints are not violated. Finally, efficacy of the proposed design is illustrated through a simulation example.
\end{abstract}

\section{Keywords}

Output regulation; internal model; Barrier Lyapunov Function; nonlinear control; tracking 


\section{Introduction}

In practice, physical systems are commonly subject to constraints leading to a necessary consideration of the transient performance of controlled systems. For example, position constraints of robot manipulators should be imposed in physical human-robot interaction design to prevent collisions with human or the surroundings (He et al. 2016a,b). Specifically, for the development of the microelectromechanical systems (MEMS), the challenging control issues (Devasia et al. 2007) imposed on the devices in nanoscale have raised a much more rigorous control performance specification. It has also promoted a wide variety of application studies, as seen in the recent work (Tee et al. 2009; Sun et al. 2015; Luo and Song 2016). The MEMS devices are quite sensitive to physical geometry constraints. For the MEMS systems with precious and fragile device, controller design without a stringent consideration on the guaranteed transient performance would greatly reduce the device lifetimes. Without a guaranteed transient performance, the movable plate may hit the fixed one again and again before coming to its steady state, which results in surface damage at each contact, as shown in Sun et al. (2012).

To deal with the constraints related to the transient performance issue, one approach is to employ the composite nonlinear feedback (CNF) technique composed of linear and nonlinear feedback parts. It was first proposed in Lin et al. (1998), and recently it has been extensively studied to improve the output regulation performance of linear

\footnotetext{
${ }^{1}$ School of Automation Science and Engineering, South China University of Technology, Guangzhou 510640, P. R. China

${ }^{2}$ Department of Aeronautical and Automotive Engineering, Loughborough University, Leicestershire, LE11 3TU, UK.
}

\section{Corresponding author:}

Yunjian Peng, School of Automation Science and Engineering, South China University of Technology, Guangzhou 510640, P. R. China

Email: pengyj@scut.edu.cn 
systems (Zhang and Lan 2013; Lin et al. 2017). In consideration of nonlinear systems with constraints, the Barrier Lyapunov Function (BLF) technique has received more and more attention (Tee et al. 2009, 2011). The BLF is not radially unbounded, but increases to infinity once its argument approaches certain finite limit (Tee et al. 2009). This implies that the finite limit can be carefully assigned to meet the actual desired constraint barriers. In order to relax the requirement of static constraint (Tee et al. 2009) and reduce design conservativeness, a time-varying BLF was used in Tee et al. (2011), which allows the expected barrier limit to vary in real time with the desired output trajectory. Up to now, the BLF-based control technique has been used for many kinds of nonlinear systems with output constraints, see He et al. (2015); Liu and Tong (2016); Li et al. (2019) and the references therein. However, most of the existing works rely on the classical inversionbased tracking design method, which requires the differential information of the tracking reference unusually unavailable in real world applications. Moreover, instead of realizing asymptotic tracking for the desired trajectories of interest, the neural network methods can only make the tracking error converge into a compact set around zero (Yang et al. 2017; Liu et al. 2018; Li et al. 2019). In contrast to the inversion-based tracking control with neural network methods, by employing the internal model principle (Huang 2004), the output regulation method can remove the requirement of the differential information of higher order whilst achieving asymptotic tracking.

In this paper, we will handle the adaptive output regulation problem with prescribed performance guarantees based on the symmetric time-varying BLF (TVBLF) technique. The main contributions are as follows. First, as an extension, we consider the class of nonlinear systems with nonlinear zero dynamics, which is more general than the output feedback systems (Sun and Huang 2009; Guo and Liu 2018). Technically, the approach in Sun and Huang (2009) and Guo and Liu (2018) cannot handle the nonlinear systems such as the generalized Lorenz system in the numerical example. To achieve this, we have applied the technique of change supply functions. Second, the system uncertain parameters belong to some known arbitrarily large compact set. To address this difficulty, a robust law is combined with the high gain technique. Third, the presented 
result contains the static output constraint problem in Sun et al. (2012) as a special case, and enlarges the set of feasible initial outputs. The proposed output feedback control law allows asymptotic convergence of the tracking error with prescribed transient performance guarantees despite the presence of uncertain parameters. Comparative simulation results demonstrate that the proposed BLF-based design in the output regulation problem is of guaranteed efficiency. Therefore, it can be expected to extend the BLF-based technique to the existing event-based or cooperative output regulation problems (Liu 2015; Liu and Huang 2017; Su et al. 2019).

Throughout this paper, $\mathbb{R}^{n}$ denotes the $n$-dimensional Euclidean space, $\|\cdot\|$ represents the induced matrix norm or the Euclidean vector norm, $\mathbb{R}_{+}$is the set of non-negative real numbers and $I_{n}$ is the $n \times n$ identity matrix. The notations $\in$ and $\subset$ used in the expression such as $w \in \mathbb{W} \subset \mathbb{R}^{n_{w}}$ indicates that $w$ is a member of $\mathbb{W}$, where $\mathbb{W}$ is a compact subset of $\mathbb{R}^{n_{w}}$. For a number of $m$ column vectors $x_{1}, \ldots, x_{m},\left(x_{1}, \ldots, x_{m}\right)^{\top}$ denotes $\left[x_{1}^{\top}, \ldots, x_{m}^{\top}\right]^{\top}$, where the superscript $\mathrm{T}$ means the transpose of a matrix or vector.

\section{Problem Formulation and Preliminaries}

Consider a class of nonlinear systems described by

$$
\begin{aligned}
& \dot{z}=f(z, y, v, w) \\
& \dot{y}=g(z, y, v, w)+b(w) u \\
& e=y-q(v, w)
\end{aligned}
$$

where $(z, y)^{T} \in \mathbb{R}^{n} \times \mathbb{R}$ is the state, $u \in \mathbb{R}$ and $y \in \mathbb{R}$ are the control input and the output, respectively, $e \in \mathbb{R}$ is the regulated output error, $w \in \mathbb{W} \subset \mathbb{R}^{n_{w}}$ represents an uncertain constant parameter vector, and $v(t) \in \mathbb{V} \subset \mathbb{R}^{n_{v}}$ represents both external disturbance and reference input. In the output regulation framework, the exogenous signal $v(t)$ is assumed to be generated by the following linear exosystem

$$
\dot{v}=S(\sigma) v, \quad y_{r}=q(v, w)
$$


where $\sigma \in \mathbb{S} \subset \mathbb{R}^{n_{\sigma}}$ represents the unknown constant parameters. All the functions in systems (1) and (2) are supposed to be sufficiently smooth and satisfy $f(0,0,0, w)=0$, $g(0,0,0, w)=0, q(0, w)=0$ for all $w \in \mathbb{R}^{n_{w}}$.

The problem studied here is stated as follows.

Problem 1. Given $\mathbb{V}, \mathbb{W}$ and $\mathbb{S}$ with $0 \in \mathbb{V} \times \mathbb{W} \times \mathbb{S}$, find a dynamic output feedback control law of the form

$$
\begin{aligned}
\dot{\eta} & =M \eta+N u \\
u & =h(\eta, \hat{\Psi}, k, e) \\
\dot{\hat{\Psi}} & =l(\eta, \hat{\Psi}, k, e) \\
\dot{k} & =\bar{\rho}(\eta, \hat{\Psi}, k, e)
\end{aligned}
$$

where $h(\cdot), l(\cdot), \bar{\rho}(\cdot)$ are some nonlinear functions and $(M, N)$ is a pair of matrices to be designed later, such that, for any $\operatorname{col}(v, w, \sigma) \in \mathbb{V} \times \mathbb{W} \times \mathbb{S}$, some specified initial state $y(0)$ in a fixed and known compact set, and any other initial states $z(0), v(0), \eta(0), \hat{\Psi}(0), k(0)$, the resulting closed-loop system composed of (1)-(3) holds the following three properties:

1) the trajectory of the closed-loop system exists and is bounded over $[0, \infty)$;

2 ) the tracking error $e(t)$ asymptotically converges to zero, i.e., $\lim _{t \rightarrow \infty} e(t)=0$;

3) the output $y(t)$ satisfies $|y(t)| \leq k_{c}(t), \forall t \geq 0$, for some prescribed time-varying constraint $k_{c}(t)$.

Remark 1. We call the aforementioned problem as the adaptive output regulation problem with prescribed performance guarantees for nonlinear systems with relative degree one. Compared with the existing results in Sun and Huang (2009) and Guo and Liu (2018), the considered system (1) can represent a larger class of nonlinear systems since it allows the zero dynamics $z$ to be nonlinear. As in Xu and Huang (2010), the tracking error should asymptotically approach zero under the unknown exosystem. An additional condition here is that the output should evolve within a desired region characterized by the time-varying function $k_{c}(t)$. 
Remark 2. In the controller (3), $\eta$ denotes the internal model dynamic (Huang 2004), $\hat{\Psi}$ is the adaptive gain to estimate the unknown $\sigma$, and $k$ is the high gain to cover the uncertain parameters. Here, the dynamics $\eta$ are in the linear form since we consider the output regulation problem with the linear exosystem (2). However, it is necessary to introduce the nonlinear internal model if the nonlinear exosystem as in Lu and Huang (2015) is considered.

For any known compact subsets $\mathbb{V} \subset \mathbb{R}^{n_{v}}$ and $\mathbb{W} \subset \mathbb{R}^{n_{w}}$, there always exists a bound $\bar{y}_{r}(t)$ of the desired trajectory $y_{r}(t)=q(v(t), w)$ for all $v(t) \in \mathbb{V}$ and $w \in \mathbb{W}$. Denote

$$
k_{b}(t)=k_{c}(t)-\bar{y}_{r}(t) .
$$

If the tracking error $e(t)$ satisfies

$$
|e(t)| \leq k_{b}(t), \forall t \geq 0
$$

with $\bar{y}_{r}(t)$ designed to have the following property

$$
\left|y_{r}(t)\right| \leq \bar{y}_{r}(t)<k_{c}(t), \forall t \geq 0,
$$

then we can guarantee satisfaction of the original output constraint in Problem 1, as will be stated in Theorem 1 .

Since the first order time derivative of $\bar{y}_{r}(t)$ is required in the subsequent design, a choice of $\bar{y}_{r}(t)$ can be chosen as a function of $y_{r}(t)$ in the following

$$
\bar{y}_{r}(t)=\left\{\begin{array}{cl}
-(2 \lambda / \pi) \cos \left(\frac{\pi y_{r}(t)}{2 \lambda}\right)+\lambda, & \left|y_{r}(t)\right| \leq \lambda \\
\left|y_{r}(t)\right|, & \left|y_{r}(t)\right|>\lambda
\end{array}\right.
$$

where $\lambda$ is any positive constant satisfying $\lambda<\sup _{t \geq 0} y_{r}(t)$. Alternatively, we can also use a simple form of $\bar{y}_{r}(t)$ as

$$
\bar{y}_{r}(t)=A_{m}
$$

where $A_{m}$ is the amplitude of the desired trajectory $y_{r}(t)$.

Moreover, we have $0<\underline{k}_{b}<k_{b}(t)<\bar{k}_{b}, \forall t \geq 0$, where $\underline{k}_{b}$ and $\bar{k}_{b}$ are positive constants given by

$$
\underline{k}_{b}=\inf _{t>0}\left\{k_{c}(t)-\bar{y}_{r}(t)\right\}, \quad \bar{k}_{b}=\sup _{t>0}\left\{k_{c}(t)-\bar{y}_{r}(t)\right\} .
$$


Remark 3. By introducing the bound $\bar{y}_{r}(t)$ of the desired trajectory, we have transformed the original constraint on output into the transient performance requirement of tracking error. This facilitates the solution to the adaptive output regulation of system (1) with the output constraint. Thanks to such a formulation, Problem 1 can be converted into an constraint adaptive stabilization problem of a so-called augmented system regarding the tracking error as the constraint output.

In the following, a dynamic compensator called internal model will be established, based on which it will be shown that with some coordinate transformation, the constrained stabilization solution of a transformed augmented system will lead to the solution of Problem 1. To this end, let us introduce some standard assumptions (Xu and Huang 2010; Liu and Huang 2017).

Assumption 1. For the unknown $\sigma \in \mathbb{S}$, all eigenvalues of the matrix $S(\sigma)$ are distinct and have zero real parts.

Assumption 2. For all $w \in \mathbb{R}^{n_{w}}$, the unknown continuous function $b(w)$ satisfies $b(w) \neq 0$ and without loss of generality, we assume $b(w)>0$.

Remark 4. Assumptions 1 and 2 are standard for solving the related output regulation problem in literature (Xu and Huang 2010; Liu and Huang 2017). Under Assumption 1, the exosystem (2) produces exogenous signal in a sum form of finitely many sinusoidal functions. The frequencies are determined by the eigenvalues of $S(\sigma)$ while the phase angles and amplitudes can be calculated according to the initial condition. By introducing the nonlinear exosystem ( $\mathrm{Lu}$ and Huang 2015), Assumption 1 can be relaxed to allow a much larger class of exogenous signals. Moreover, Assumption 2 loses no generality as remarked in Liu and Huang (2017). In fact, the only requirement is $b(w) \neq 0$ for all $w \in \mathbb{R}^{n_{w}}$. For the case where the sign of the control gain $b(w)$ is unknown, the Nussbaum gain technique can be employed (Xu and Huang 2009; Guo and Liu 2018).

Assumption 3. There exists a sufficiently smooth function $\mathbf{z}: \mathbb{R}^{n_{v}} \times \mathbb{R}^{n_{w}} \times \mathbb{R}^{n_{\sigma}} \mapsto$ $\mathbb{R}^{n}$ defined globally with $\mathbf{z}(0, w, \sigma)=0$ such that

$$
\frac{\partial \mathbf{z}(v, w, \sigma)}{\partial v} S(\sigma) v=f(\mathbf{z}(v, w, \sigma), q(v, w), v, w)
$$


for any $(v, w, \sigma) \in \mathbb{R}^{n_{v}} \times \mathbb{R}^{n_{w}} \times \mathbb{R}^{n_{\sigma}}$.

Under Assumption 3, let $\mathbf{y}(v, w, \sigma)=q(v, w)$. It can be obtained that

$$
\begin{aligned}
\mathbf{u}(v, w, \sigma)= & b^{-1}(w)[(\partial q(v, w) / \partial v) S(\sigma) v \\
& -g(\mathbf{z}(v, w, \sigma), q(v, w), v, w)],
\end{aligned}
$$

and consequently $\mathbf{z}(v, w, \sigma), \mathbf{y}(v, w, \sigma)$ and $\mathbf{u}(v, w, \sigma)$ give the solution of the defined regulator equations (Isidori and Byrnes 1990; Huang 2004) related to (1) and (2).

Due to the explicit dependency on the external signal $v$ and uncertain parameter $(w, \sigma)$, the solution $\mathbf{u}(v, w, \sigma)$ providing the feedforward control knowledge cannot be directly applied to solve the output regulation problem. Technically, an internal model in the form of dynamic compensator (Huang 2004) should be constructed to provide the necessary information of $\mathbf{u}(v, w, \sigma)$. To ensure the existence of such an internal model, we need a further assumption.

Assumption 4. The solution $\mathbf{u}(v, w, \sigma)$ is a polynomial function in $v$ with its coefficients depending on $w$ and $\sigma$.

Remark 5. Assumption 3 is a necessary condition for the solvability of the output regulation problem (Isidori and Byrnes 1990). The solution to the regulator equation (10) has been studied in Huang $(2003,2004)$. Note that, for the case where the exosystem (2) is linear, the solution to (10) can be guaranteed if the function $f(z, y, v, w)$ in (1) is polynomial in $(z, y, v)$ with coefficients depending on $w$ (Huang 2003, 2004). Moreover, Assumption 4 is satisfied automatically if the functions $q(v, w)$ and $g(z, y, v, w)$ in (1) have the same polynomial feature in $(z, y, v)$ as $f(z, y, v, w)$.

As remarked in $\mathrm{Xu}$ and Huang (2010), under Assumption 4, there exists a set of real numbers $a_{1}(\sigma), a_{2}(\sigma), \ldots, a_{s}(\sigma)$ for some positive integer $s$, such that, the roots of the following real coefficient polynomial

$$
P^{\sigma}(\lambda)=\lambda^{s}-a_{1}(\sigma)-a_{2}(\sigma) \lambda-\ldots-a_{s}(\sigma) \lambda^{s-1}
$$


are all distinct with zero real part for any $\sigma \in \mathbb{S}$, and meanwhile $\mathbf{u}(v, w, \sigma)$ satisfies

$$
\begin{aligned}
\frac{d^{s} \mathbf{u}(v, w, \sigma)}{d t^{s}}= & a_{1}(\sigma) \mathbf{u}(v, w, \sigma)+a_{2}(\sigma) \frac{d \mathbf{u}(v, w, \sigma)}{d t} \\
& +\cdots+a_{s}(\sigma) \frac{d^{s-1} \mathbf{u}(v, w, \sigma)}{d t^{s-1}}
\end{aligned}
$$

for all $v$ related to the exosystem (2) and any $(w, \sigma) \in \mathbb{W} \times \mathbb{S}$.

Define

$$
\begin{aligned}
\Phi(\sigma) & =\left[\begin{array}{cc}
0_{(s-1) \times 1} & I_{s-1} \\
a_{1}(\sigma) & a_{2}(\sigma) \cdots a_{s}(\sigma)
\end{array}\right], \\
\Gamma & =\left[\begin{array}{c}
1 \\
0_{(s-1) \times 1}
\end{array}\right]^{T} .
\end{aligned}
$$

Choose a Hurwitz matrix $M \in \mathbb{R}^{s \times s}$ and a column vector $N \in \mathbb{R}^{s \times 1}$ such that $(M, N)$ is a controllable pair. Since for each $\sigma \in \mathbb{S}$, the pair $(\Gamma, \Phi(\sigma))$ is observable with the eigenvalues of $\Phi(\sigma)$ having zero real part, there is a unique and nonsingular matrix $T(\sigma)$ satisfying the Sylvester equation (Nikiforov 1998)

$$
T(\sigma) \Phi(\sigma)-M T(\sigma)=N \Gamma
$$

Denote $\quad \theta(v, w, \sigma)=T(\sigma) \tau(v, w, \sigma) \quad$ where $\quad \tau(v, w, \sigma)=$ $\left(\mathbf{u}(v, w, \sigma), \dot{\mathbf{u}}(v, w, \sigma), \ldots, \mathbf{u}^{s-1}(v, w, \sigma)\right)^{\top}$, and let $\Psi^{\sigma}=\Gamma T^{-1}(\sigma)$. Then, we can verify that $\theta(v, w, \sigma)$ satisfies

$$
\begin{aligned}
\frac{\partial \theta(v, w, \sigma)}{\partial v} S(\sigma) v & =\left(M+N \Psi^{\sigma}\right) \theta(v, w, \sigma) \\
\mathbf{u}(v, w, \sigma) & =\Psi^{\sigma} \theta(v, w, \sigma)
\end{aligned}
$$

and therefore, on the basis of (15), a dynamic compensator called the internal model of the composite system (1) and (2) with output $u$ can be established as follows

$$
\dot{\eta}=M \eta+N u
$$

Remark 6. The establishment of the internal model dynamics (16) follows from the same procedure in Huang and Chen (2004). The existence of the internal model for 
various nonlinear systems have gained extensive research in the past decades, see Nikiforov (1998); Lu and Huang (2015) and the references therein. In particular, it is shown in Liu et al. (2009) that if Assumption 4 is satisfied and the exosystem (2) is linear, then there exists an internal model of the form (16). From (15) and (16), it is known that the internal model is used to asymptotically generate the function $\theta(v, w, \sigma)$ such that the unavailable information of $\mathbf{u}(v, w, \sigma)$ can be reproduced asymptotically (Liu et al. 2009).

In this respect, we can transform Problem 1 into the constrained stabilization problem of an augmented system. For this purpose, performing on the system composed of (1) and (16) the following coordinate transformation

$$
\begin{aligned}
& \bar{z}=z-\mathbf{z}(v, w, \sigma) \\
& \bar{\eta}=\eta-\theta(v, w, \sigma)-N b^{-1} e \\
& e=y-q(v, w)
\end{aligned}
$$

yields

$$
\begin{aligned}
& \dot{\bar{z}}=\bar{f}(\bar{z}, e, \mu) \\
& \dot{\bar{\eta}}=M \bar{\eta}+M N b^{-1} e-N b^{-1} \bar{g}(\bar{z}, e, \mu) \\
& \dot{e}=\bar{g}(\bar{z}, e, \mu)+b \Psi^{\sigma} \bar{\eta}+\Psi^{\sigma} N e+b \bar{u}
\end{aligned}
$$

where $\mu=(v, w, \sigma), b=b(w), \bar{u}=u-\Psi^{\sigma} \eta$ and

$$
\begin{aligned}
\bar{f}(\bar{z}, e, \mu)= & f(\bar{z}+\mathbf{z}(v, w, \sigma), e+q(v, w), v, w) \\
& -f(\mathbf{z}(v, w, \sigma), q(v, w), v, w) \\
\bar{g}(\bar{z}, e, \mu)= & g(\bar{z}+\mathbf{z}(v, w, \sigma), e+q(v, w), v, w) \\
& -g(\mathbf{z}(v, w, \sigma), q(v, w), v, w) .
\end{aligned}
$$

For any $\mu \in \mathbb{R}^{n_{v}} \times \mathbb{W} \times \mathbb{S}$, it can be obtained that $\bar{f}(0,0, \mu)=0$ and $\bar{g}(0,0, \mu)=0$.

Remark 7. For system (18), we consider $e(t)$ as the controlled output under the unknown disturbance $\mu \in \mathbb{V} \times \mathbb{W} \times \mathbb{S}$ in the compact sets. If we can design a controller 
in form of

$$
\bar{u}=h(\eta, \hat{\Psi}, k, e), \quad \dot{\hat{\Psi}}=l(\eta, \hat{\Psi}, k, e), \quad \dot{k}=\bar{\rho}(\eta, \hat{\Psi}, k, e)
$$

solving the constrained stabilization problem of (18) in the sense that, for the specified initial states $e(0)$ and any other initial states $\bar{z}(0), v(0), \bar{\eta}(0), \hat{\Psi}(0), k(0)$ of the closedloop system composed of (18) and (19), the trajectory of the closed-loop system exists and is bounded for all $t \geq 0$, and the state of the augmented system (18) asymptotically converges to zero with $e(t)$ satisfying (5), then the control law (3) solves Problem 1. In contrast to solving the robust stabilization problem of the augmented system (18) as seen in $\mathrm{Xu}$ and Huang (2010), a much more rigorous issue is proposed, where the specific transformed constrained stabilization problem here requires that the constrained augmented system state $e(t)$ should satisfy $|e(t)| \leq k_{b}(t)$ with some specified timevarying function $k_{b}(t)$ while approaching zero.

\section{Main Result}

In what follows, we will handle the constrained stabilization problem of (18) by employing the BLF technique. To this end, the following lemma and assumption adopted from (Tee et al. 2009) and (Xu and Huang 2010), respectively, are listed.

Lemma 1. Let $\mathcal{Z}=\{\xi \in \mathbb{R}:|\xi|<1\} \subset \mathbb{R}$ and $\mathbb{N}=\mathbb{R}^{l} \times \mathcal{Z} \subset \mathbb{R}^{l+1}$ be open sets. Consider the system

$$
\dot{\eta}=h(t, \eta)
$$

where $\eta=(w, \xi)^{T} \in \mathbb{N}$, and $h: \mathbb{R}_{+} \times \mathbb{N} \rightarrow \mathbb{R}^{l+1}$ is piecewise continuous in $t$ and locally Lipschitz in $\eta$, uniformly in $t$, on $\mathbb{R}_{+} \times \mathbb{N}$. Suppose that there exist functions $U: \mathbb{R}^{l} \times \mathbb{R}_{+} \rightarrow \mathbb{R}_{+}$and $V_{1}: \mathcal{Z} \rightarrow \mathbb{R}_{+}$, continuously differentiable and positive definite in their respective domains, such that

$$
\begin{array}{r}
V_{1}(\xi) \rightarrow \infty \quad \text { as } \quad|\xi| \rightarrow 1 \\
\gamma_{1}(\|w\|) \leq U(w, t) \leq \gamma_{2}(\|w\|)
\end{array}
$$


where $\gamma_{1}$ and $\gamma_{2}$ are class $K_{\infty}$ functions. Let $V(\eta)=V_{1}(\xi)+U(w, t)$ and $\xi(0) \in \mathcal{Z}$. If the following inequality holds

$$
\dot{V}=\frac{\partial V}{\partial \eta} h \leq 0
$$

in the set $\xi \in \mathcal{Z}$, then $\xi(t) \in \mathcal{Z}, \forall t \in[0, \infty)$.

Assumption 5. For any compact subset $\Sigma \subset \mathbb{R}^{n_{v}} \times \mathbb{W} \times \mathbb{S}$, there exists a $C^{1}$ function $V(\bar{z})$ satisfying

$$
\begin{gathered}
\underline{\alpha}(\|\bar{z}\|) \leq V(\bar{z}) \leq \bar{\alpha}(\|\bar{z}\|) \\
\frac{\partial V(\bar{z})}{\partial \bar{z}} \bar{f}(\bar{z}, e, \mu) \leq-\alpha(\|\bar{z}\|)+\delta \gamma(e)
\end{gathered}
$$

for system (18) with $\mu \in \Sigma$, where $\underline{\alpha}(\cdot)$ and $\bar{\alpha}(\cdot)$ are some class $\mathcal{K}_{\infty}$ functions, $\delta$ is some unknown positive constant, $\gamma(\cdot)$ is a known positive definite smooth function, and $\alpha(\cdot)$ is a known class $\mathcal{K}_{\infty}$ function satisfying $\lim _{s \rightarrow 0^{+}} \sup \left(\alpha^{-1}\left(s^{2}\right) / s\right)<\infty$.

Denote $Z=\operatorname{col}(\overline{\mathrm{z}}, \bar{\eta})$. Regarding $e$ as the output, we have the zero dynamics of system (18) given by the $Z$ subsystem. It can be seen that the zero dynamics is inherent nonlinear. However, Assumption 5 shows that the $\bar{z}$ subsystem of system (18) is input-to-state stable for any $\mu \in \Sigma$ viewing $e$ as the input and $\bar{z}$ as the state. Furthermore, notice that the $(\bar{z}, \bar{\eta})$ subsystem is in the form of eq. (17) in Xu and Huang (2010), where $z_{1}=\bar{z}, z_{2}=\bar{\eta}, u=e, \varphi_{1}=\bar{f}(\bar{z}, e, \mu), A=M$, and $\varphi_{2}=M N b^{-1} e-N b^{-1} \bar{g}(\bar{z}, e, \mu)$. Therefore, according to Lemma 3.1 in $\mathrm{Xu}$ and Huang (2010), there exists a $C^{1}$ function $V_{1}(\bar{z}, \bar{\eta})$, for any $\mu \in \Sigma$, satisfying

$$
\underline{\alpha}_{1}(\|\bar{z}, \bar{\eta}\|) \leq V_{1}(\bar{z}, \bar{\eta}) \leq \bar{\alpha}_{1}(\|\bar{z}, \bar{\eta}\|)
$$

for some class $\mathcal{K}_{\infty}$ functions $\underline{\alpha}_{1}(\cdot)$ and $\bar{\alpha}_{1}(\cdot)$, such that, along the trajectory of $(\bar{z}, \bar{\eta})$ subsystem of (18)

$$
\dot{V}_{1} \leq-\|\bar{z}\|^{2}-\|\bar{\eta}\|^{2}+\delta_{e} \gamma_{e}(e)
$$

for some positive constant $\delta_{e}$ and some known positive definite function $\gamma_{e}(\cdot)$.

Remark 8. We can consider the augmented system (18) as in the lower triangular form by viewing the $Z$ subsystem as the dynamic uncertainty (Liu and Jiang 2016). Since 
the state $(\bar{z}, \bar{\eta})$ contains the unknown parameters $w$ and $\sigma$ as well as the unavailable signal $v$, it cannot be used for feedback control. However, as shown in (22), the $(\bar{z}, \bar{\eta})$ subsystem is input-to-state stable for any $\mu$ with respect to input $e$ and state $(\bar{z}, \bar{\eta})$. Thus, this dynamic uncertainty will not impose any particular difficulty on the stabilization solution of system (18). In such case, it is feasible to stabilize (18) without using the state $(\bar{z}, \bar{\eta})$.

Now we are ready to solve Problem 1. We first establish the following lemma solving the constrained stabilization problem of system (18). Since the dependence of exosystem (2) on unknown parameter $\sigma$ leads to the unavailable measurement of $\Psi^{\sigma} \eta$, we would incorporate the adaptive control method into our design scheme.

Lemma 2. Under Assumption 5, the feedback control law

$$
\begin{aligned}
u & =-\frac{k_{b}^{2}-e^{2}}{2} k \rho(e) e+\hat{\Psi} \eta \\
\dot{\hat{\Psi}} & =-\frac{2 \eta^{T} e}{k_{b}^{2}-e^{2}} \\
\dot{k} & =\rho(e) e^{2}
\end{aligned}
$$

solves the constrained stabilization problem of system (18) in the sense given in Remak 7. In (23), $\hat{\Psi}$ is the estimation of $\Psi^{\sigma}, k$ is the high gain dynamically generated to cover the uncertainties $\mu$, and $\rho(e)$ is some determined smooth positive function.

Proof: Notice the existence of (22). By applying the changing supply functions technique (Sontag and Teel 1995), for any positive definite smooth function $\Delta(Z)>0$, we can obtain a $C^{1}$ function $V_{Z}(Z)$ satisfying

$$
\underline{\alpha}_{2}(\|\bar{z}, \bar{\eta}\|) \leq V_{Z}(Z) \leq \bar{\alpha}_{2}(\|\bar{z}, \bar{\eta}\|)
$$

for some class $\mathcal{K}_{\infty}$ functions $\underline{\alpha}_{2}(\cdot)$ and $\bar{\alpha}_{2}(\cdot)$, such that

$$
\dot{V}_{Z} \leq-\Delta(Z)\|Z\|^{2}+\bar{\delta}_{e} \bar{\gamma}_{e}(e) e^{2}
$$

for some positive constant $\bar{\delta}_{e}$ and some known positive function $\bar{\gamma}_{e}(\cdot)$. 
Let

$$
V_{b l f}=\log \frac{k_{b}^{2}(t)}{k_{b}^{2}(t)-e^{2}(t)}
$$

where $\log (\bullet)$ is the natural $\log$ arithm of $\bullet$.

By the change of error coordinates $\xi=\frac{e(t)}{k_{b}(t)}$, we can rewrite $V_{b l f}$ into $V_{b l f}=$ $\log \frac{1}{1-\xi^{2}}$. It is clear that, in the set $|\xi|<1, V_{b l f}$ is continuously differentiable and positive definite. Then, the time derivative of $V_{b l f}$ along the $e$ subsystem of system (18) under the control law (23) satisfies

$$
\begin{aligned}
\dot{V}_{b l f}= & \frac{2 \xi}{k_{b}\left(1-\xi^{2}\right)}\left(\dot{e}-e \frac{\dot{k}_{b}}{k_{b}}\right) \\
= & \frac{2 e}{k_{b}^{2}-e^{2}}\left(\tilde{g}(\bar{z}, \bar{\eta}, e, \mu)-e \frac{\dot{k}_{b}}{k_{b}}\right)-b k \rho(e) e^{2} \\
& -\frac{2 e}{k_{b}^{2}-e^{2}} b\left(\Psi^{\sigma}-\hat{\Psi}(t)\right) \eta
\end{aligned}
$$

where $\tilde{g}(\bar{z}, \bar{\eta}, e, \mu)=\bar{g}(\bar{z}, e, \mu)+b \Psi^{\sigma} \bar{\eta}+\Psi^{\sigma} N e$.

Since $\tilde{g}(\bar{z}, \bar{\eta}, e, \mu)$ is smooth with $\tilde{g}(0,0,0, \mu)=0$ for any $\mu \in \Sigma$, using Lemma 7.8 in Huang (2004) gives

$$
\left|\bar{g}_{e}(\bar{z}, \bar{\eta}, e, \mu)\right|^{2} \leq \kappa\left(\varphi(Z)\|Z\|^{2}+\pi(e)\|e\|^{2}\right)
$$

where $\kappa$ is a positive constant, $\varphi(Z)$ and $\pi(e)$ are two smooth positive functions.

Denote $\tilde{\Psi}(t)=\Psi^{\sigma}-\hat{\Psi}(t)$. Let

$$
U=V_{Z}(Z)+V_{b l f}+\frac{1}{2} b(k-\bar{k})^{2}+\frac{1}{2} b \tilde{\Psi}(t) \tilde{\Psi}(t)^{\top}
$$

where $\bar{k}$ is a positive constant number to be specified later. Using inequalities (25) and (28), we can calculate the derivative of $U$ along the closed-loop system composed of (18) 
and (23)

$$
\begin{aligned}
\dot{U} \leq & -\Delta(Z)\|Z\|^{2}+\bar{\delta}_{e} \bar{\gamma}_{e}(e) e^{2}+\frac{e^{2}}{\left(k_{b}^{2}-e^{2}\right)^{2}} \\
& +\kappa\left(\varphi(Z)\|Z\|^{2}+\pi(e)\|e\|^{2}\right)+\left(\frac{\dot{k}_{b}}{k_{b}}\right)^{2} e^{2} \\
& -b k \rho(e) e^{2}-\frac{2 e}{k_{b}^{2}-e^{2}} b \tilde{\Psi} \eta+b \dot{k}(k-\bar{k})-b \dot{\hat{\Psi}} \tilde{\Psi}^{\top} \\
\leq & -(\Delta(Z)-\kappa \varphi(Z))\|Z\|^{2}+\left(\bar{\delta}_{e} \bar{\gamma}_{e}(e)+\frac{1}{\left(k_{b}^{2}-e^{2}\right)^{2}}\right. \\
& \left.+\kappa \pi(e)+\left(\frac{\dot{k}_{b}}{k_{b}}\right)^{2}\right) e^{2}-b k \rho(e) e^{2}+b \dot{k}(k-\bar{k}) \\
& -b\left(\dot{\hat{\Psi}}+\frac{2 \eta^{T} e}{k_{b}^{2}-e^{2}}\right) \tilde{\Psi}^{\top} \\
\leq & -(\Delta(Z)-\kappa \varphi(Z))\|Z\|^{2}+\left(\iota\left(e, k_{b}\right)-b \bar{k} \rho(e)\right) e^{2}
\end{aligned}
$$

where $\iota\left(e, k_{b}\right)=\bar{\delta}_{e} \bar{\gamma}_{e}(e)+\frac{1}{\left(k_{b}^{2}-e^{2}\right)^{2}}+\kappa \pi(e)+\left(\frac{\dot{k}_{b}}{k_{b}}\right)^{2}$.

Choosing smooth functions $\Delta(Z)$ and $\rho(e)$ with

$$
\begin{aligned}
\Delta(Z) & \geq \kappa \varphi(Z)+1 \\
\rho(e) & \geq \max \left\{\bar{\gamma}_{e}(e), \pi(e), 1\right\}+\frac{1}{\left(k_{b}^{2}-e^{2}\right)^{2}}
\end{aligned}
$$

and letting $\bar{k}$ be such that $\bar{k} \geq b^{-1}\left(\bar{\delta}_{e}+\kappa+\left(\frac{\dot{k}_{b}}{k_{b}}\right)^{2}\right)$, we have

$$
\dot{U} \leq-\|Z\|^{2} \text {. }
$$

Therefore, the function $U$ given by (29) satisfies (30) for the closed-loop system composed of (18) and (23).

Define $\bar{x}_{c}=\operatorname{col}(\bar{z}, \bar{\eta}, e, \hat{\Psi}, k)$. Since $U$ is positive definite with its derivative satisfying (30), it can be concluded that $\bar{x}_{c}$ is bounded for all $t \geq 0$, and $\lim _{t \rightarrow \infty}(\|\bar{z}\|+\|\bar{\eta}\|)=$ 0 . Note that the uncertain parameters $v, w$ and $\sigma$ are bounded. By the coordinate transformation in (17), $\eta$ is bounded. Therefore, for the composite system composed of (18) and (23), all the states with their derivatives are bounded. Meanwhile, it can be seen that $\dot{k}=\rho(e) e^{2}$ is bounded due to the boundedness of $e(t)$. Moreover, $\dot{k}$ is uniformly continuous since $\dot{e}(t)$ is also bounded. By Barbalat's lemma, $\dot{k}=\rho(e) e^{2}$ approaches zero 
as $t \rightarrow+\infty$, leading to $\lim _{t \rightarrow \infty} e(t)=0$. Since $\lim _{|e| \rightarrow k_{b}} \log \frac{k_{b}^{2}}{k_{b}^{2}-e^{2}}=\infty$, then using Lemma 1 , we obtain $|e(t)| \leq k_{b}(t)$ if the initial state of $e(t)$ belongs to a prescribed region, i.e., $|e(0)| \leq k_{b}(0)$. Finally, the controller (23) solves the constrained stabilization problem for system (18). The proof is completed.

Using Lemma 2 and Remark 7 gives our main result as follows.

Theorem 1. Under Assumptions 1-4, Problem 1 is solvable for system (1) by the feedback controller

$$
\begin{aligned}
& u=-\frac{k_{b}^{2}-e^{2}}{2} k \rho(e) e+\hat{\Psi} \eta \\
& \dot{\eta}=M \eta+N u \\
& \dot{\hat{\Psi}}=-\frac{2 \eta^{T} e}{k_{b}^{2}-e^{2}} \\
& \dot{k}=\rho(e) e^{2}
\end{aligned}
$$

where $\rho(e)$ is the same as in (23).

Proof: It is easy to verify Properties 1) and 2) in Problem 1 by Lemma 2 and Remak 7. We will now consider the satisfaction of Property 3) which shows that the constraint of the output $y(t)$ is never violated if the initial error output $e(0)$ satisfies

$$
|e(0)|<k_{b}(0)
$$

From (30), it is clear that $U(t) \leq U(0), \forall t>0$. Together with the expression of $U(t)$ in (29), we have

$$
\log \frac{k_{b}^{2}(t)}{k_{b}^{2}(t)-e^{2}(t)} \leq U(0)
$$

which implies that $k_{b}^{2}(t) \leq e^{U(0)}\left(k_{b}^{2}(t)-e^{2}(t)\right)$. Thus

$$
|e(t)| \leq k_{b}(t) \sqrt{1-e^{-U(0)}} \leq k_{b}(t), \quad \forall t>0
$$

From the fact that $y(t)=e(t)+y_{r}(t)$ as well as the definitions in (4) and (6), we can obtain

$$
|y(t)| \leq|e(t)|+\left|y_{r}(t)\right| \leq k_{b}(t)+\bar{y}_{r}(t)=k_{c}(t)
$$


Therefore, we conclude that Property 3) is satisfied. This completes the proof.

Remark 9. Concerned with the convergence of parameter $\hat{\Psi}$ to $\Psi^{\sigma}$, an output regulation controller containing the minimal internal model should be employed (Liu et al. 2009). By Theorem 4.1 in Liu et al. (2009), if the internal model (16) of minimal dimension is used, then the feedback controller (31) would lead to $\lim _{t \rightarrow \infty}\left(\hat{\Psi}-\Psi^{\sigma}\right)=0$. For more details of determining the minimal internal model, one can refer to the work in Liu et al. (2009).

Should $\Psi^{\sigma} \eta$ be known, there is no need to introduce $\hat{\Psi}$, and consequently we can set the dimension of $\hat{\Psi}$ in (31) to zero.

Corollary 1. Under Assumptions 1-4, if the parameter $\sigma$ in (2) is known, then the following controller

$$
u=-\frac{k_{b}^{2}-e^{2}}{2} k \rho(e) e+\Psi^{\sigma} \eta, \quad \dot{k}=\rho(e) e^{2}
$$

solves the constrained stabilization problem of system (18). As a result, the feedback controller

$$
\begin{aligned}
& u=-\frac{k_{b}^{2}-e^{2}}{2} k \rho(e) e+\Psi^{\sigma} \eta \\
& \dot{\eta}=M \eta+N u \\
& \dot{k}=\rho(e) e^{2}
\end{aligned}
$$

solves Problem 1.

Remark 10. In controller (37), the dynamic $k$ is introduced to produce a high gain to cover any bounded disturbance $\mu$ in some unknown compact subset. This adaptive control technique called self-tuning regulator has been investigated in Kristic et al. (1995). However, if there is noise in practical applications, the dynamic $k$ may result in infinity because its derivative, as seen in (37), is always non-negative. To avoid this issue, we can select $k$ as some sufficiently large number based on the boundary knowledge of the compact subset. In this case, the controller in (36) and (37) can be simplified by directly setting $k$ to be some positive number. 


\section{Discussions about Initial Condition Requirement and Control}

\section{Signal}

\section{Initial Condition Requirements of BLF and QLF}

In the above section, by employing the BLF technique in the output regulation design, we have established an elegant result to rigorously guarantee the transient performance imposed on the controlled output. However, by appropriately choosing the adjustable controller parameters in the QLF-based design, it is also possible to ensure constraint satisfaction for given initial conditions of output.

In this subsection, we would compare the initial output requirements for the BLFbased and QLF-based designs. First, we consider the output regulation deign based on the BLF technique. According to the proof in Theorem 1, if the initial error output satisfies (32), then we have

$$
|e(t)|<k_{b}(t) .
$$

In fact, we have (34) which shows that $e(t)$ is in a subset of (38), i.e.,

$$
D=\left\{e(t) \in \mathbb{R}:|e(t)| \leqslant k_{b}(t) \sqrt{1-e^{-U_{0}}}\right\}, \forall t>0
$$

However, for assuring the constraint, the requirement of initial error output is much more rigorous when we employ QLF. In contrast to the BLF-based design, we would replace $V_{b l f}$ with $V_{q l f}=\vartheta e^{2}$ and for generality, we define $\vartheta$ as any positive constant. Let

$$
U=V_{Z}(Z)+\vartheta e^{2}+\frac{1}{2} b(k-\bar{k})^{2}+\frac{1}{2} b \tilde{\Psi}(t) \tilde{\Psi}(t)^{T}
$$

Following the same procedure in Lemma 2 and Theorem 1, we can show that the obtained output regulation controller takes the same form of (31) except that $u=-k \rho(e) e+\hat{\Psi} \eta$ and $\dot{\hat{\Psi}}=-2 \eta^{T} e$. Also, we have

$$
\dot{U} \leqslant-\|\bar{z}\|^{2}-\|\bar{\eta}\|^{2}
$$

which implies $U(t) \leqslant U(0)$. According to (39) and using the condition $0<b_{\min } \leq$ $b(w) \leq b_{\max }$, we can conclude that $U(0)$ satisfies $U(0) \leqslant \bar{U}$, where $\bar{U}$ is defined as 
the upper bound of $U(0)$ calculated by

$$
\begin{aligned}
\bar{U}= & V_{Z}(Z(0))+\vartheta e(0)^{2}+b_{\max }\left(k^{2}(0)+\bar{k}^{2}\right) \\
& +b_{\max }\left(\|\hat{\Psi}(0)\|^{2}+\left\|\Psi^{\sigma}\right\|^{2}\right) \\
= & M_{0}+\vartheta e(0)^{2}
\end{aligned}
$$

with

$$
\begin{aligned}
M_{0}= & V_{Z}(Z(0))+b_{\max }\left(k^{2}(0)+\bar{k}^{2}\right) \\
& +b_{\max }\left(\|\hat{\Psi}(0)\|^{2}+\left\|\Psi^{\sigma}\right\|^{2}\right) .
\end{aligned}
$$

Since $U(t) \leqslant \bar{U}$, we obtain $|e(t)| \leqslant \sqrt{\bar{U} / \vartheta}$. Thus, a sufficient condition to assure $|e(t)|<k_{b}(t)$ is $\sqrt{\bar{U} / \vartheta}<k_{b}(t)$. By (40), we know that the initial condition for $e(t)$ to satisfy the error output constraint is given by

$$
|e(0)|<\sqrt{k_{b}^{2}(t)-M_{0} / \vartheta}, \quad \forall t>0 .
$$

which depends on the minimum value $\underline{k}_{b}$ of $k_{b}(t)$.

Because $M_{0}>0$, it is obvious that this initial condition requirement is more stringent and it makes the transient performance guarantees of controlled output hard (even impossible) to be achieved. Notice that the condition (41) is satisfied under the additional condition $k_{b}^{2}(t)-M_{0} / \vartheta>0, \forall t>0$. Specifically, if $k_{b}(t)$ is monotone decreasing, then we have $\underline{k}_{b}<k_{b}(t)<k_{b}(0)$ such that (41) becomes

$$
|e(0)|<\sqrt{\underline{k}_{b}-M_{0} / \vartheta}, \quad \forall t>0
$$

For this case, even if the extra required condition $\underline{k}_{b}-M_{0} / \vartheta>0$ is satisfied, the possible initial conditions (42) may be more restricted than (32) for the BLF-based deign.

\section{Initial Condition Requirements of TVBLF and TIBLF}

It is also of interest to compare the proposed time-varying BLF (TVBLF) design with the time-invariant BLF (TIBLF) that employs a constant $k_{c}(t)$ for all $t>0$. Instead of defining $\bar{y}_{r}(t)$ with respect to the allowed constant bound of $y_{r}(t)$ under the worst case, i.e. $\left(\max _{t \geq 0}\left\{\left|y_{r}(t)\right|\right\}\right)$, it is allowed to vary with the desired output trajectory $y_{r}(t)$ in 
time in (7). This helps to improve the result in Sun et al. (2012) by enlarging the allowed set of feasible initial controlled output from

$$
|e(0)|<k_{c}-\max _{t \geq 0}\left\{\left|y_{r}(t)\right|\right\}
$$

to

$$
|e(0)|<k_{c}-\bar{y}_{r}(0)
$$

where $\bar{y}_{r}(0) \leq \max _{t \geq 0}\left\{\left|y_{r}(t)\right|\right\}$ as can be seen from (7).

\section{Range of Control Signal}

From the feedback controller (31), we observe that the component $1 /\left(k_{b}^{2}-e^{2}\right)$ is responsible for resisting constraint transgression. Whenever $e(t)$ approaches the specified barrier $\pm k_{b}(t)$, the aforementioned component will increase rapidly and produce a large control effort to pull $e(t)$ away from the barrier.

Unfortunately, there is a practical concern that, as the term $1 /\left(k_{b}^{2}-e^{2}\right)$ becomes large, the control input $u(t)$ may also become large resulting in the input saturation problem. However, from Lemma 2, we have theoretically known that the control signal is bounded and continuous. And we may further carefully design the control parameters such that the control signal can be limited within an acceptable range. By (34), we can in fact select the optional parameters in $U(0)$ such that $e(t)$ can be made away from $k_{b}(t)$ as much as possible leading to more relaxed control effort eventually.

There is a compromise between the available control action $u(t)$ and the specified transient performance because less control effort will naturally slow down the evolution of repelling $e(t)$ from the expected barrier. In summary, we have to make a tradeoff between the allowed control signal and the convergence performance of the closed-loop system. 


\section{Numerical Example}

Consider the generalized Lorenz system described by

$$
\begin{aligned}
\dot{z}_{1} & =a_{1} z_{1}+a_{2} y \\
\dot{z}_{2} & =a_{3} z_{2}+z_{1} y \\
\dot{y} & =b u+a_{4} z_{1}+a_{5} y-z_{1} z_{2} \\
e & =y-v_{1}
\end{aligned}
$$

where $\Omega \triangleq \operatorname{col}\left(a_{1}, a_{2}, a_{3}, a_{4}, a_{5}, b\right)$ is a parameter vector with uncertain constant values satisfying $a_{1}, a_{3}<0$ and $b>0$.

Let $\bar{\Omega} \triangleq \operatorname{col}\left(\bar{a}_{1}, \bar{a}_{2}, \bar{a}_{3}, \bar{a}_{4}, \bar{a}_{5}, \bar{b}\right)$ be the nominal value of the parameter vector, then $\Omega=\bar{\Omega}+w$, where $w \in \mathbb{W} \subset \mathbb{R}^{6}$ represents the parameter deviation from the nominal value. The desired output $v_{1}$ is generated by an external system in the form of (2) with $v=\left(v_{1}, v_{2}\right)^{\top}, v(0)=(9,0)^{\top}$ and $S(\sigma)=\left[\begin{array}{cc}0 & \sigma \\ -\sigma & 0\end{array}\right]$. Thus, Assumptions 1 and 2 are satisfied.

The objective is to design the feedback controller $u$ for system (43) such that the output $y(t)$ asymptotically tracks the desired trajectory $v_{1}(t)$, i.e., $\lim _{t \rightarrow \infty} e(t)=0$, subject to the following output constraint

$$
|y(t)|<k_{c}(t)=9.4+0.33 \cos (t), \quad \forall t>0 .
$$

We construct $\bar{y}_{r}(t)$ based on (8), and then obtain $k_{b}(t)$ according to the knowledge of $k_{c}(t)$ and $\bar{y}_{r}(t)$ as follows

$$
k_{b}(t)=9.4+0.33 \cos (t)-A_{m}
$$

where $A_{m}$ is the amplitude of the desired trajectory $v_{1}(t)$.

Like in $\mathrm{Xu}$ and Huang (2010), we can conclude that Assumption 3 is satisfied such that the solution of regulator equations can be obtained as $\left(\mathbf{z}_{1}(v, w, \sigma), \mathbf{z}_{2}(v, w, \sigma)\right)$ and $\mathbf{u}(v, w, \sigma)$. Moreover, Assumption 4 is satisfied and it can be verified that

$$
\frac{d^{4} \mathbf{u}(v, w, \sigma)}{d t^{4}}+10 \sigma^{2} \frac{d^{2} \mathbf{u}(v, w, \sigma)}{d t^{2}}+9 \sigma^{4} \mathbf{u}(v, w, \sigma)=0 .
$$


Hence, we have

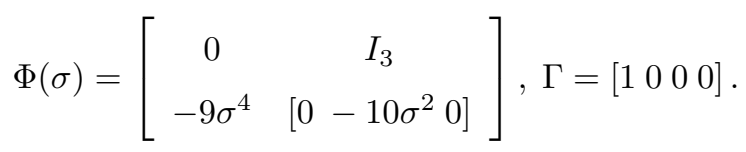

Denoting any controllable pair $(M, N)$ of the form

$$
\begin{aligned}
& M=\left[\begin{array}{cc}
0 & I_{3} \\
-m_{1} & {\left[\begin{array}{ll}
-m_{2} & -m_{3}-m_{3}
\end{array}\right]}
\end{array}\right], \\
& N=(0,0,0,1)^{\top}
\end{aligned}
$$

where $m_{1}, m_{2}, m_{3}, m_{4}>0$, we can obtain the internal model

$$
\dot{\eta}=M \eta+N u
$$

Solving the Sylvester equation (14) with $\left(m_{1}, m_{2}, m_{3}, m_{4}\right)=(4,12,13,6)$ leads to

$$
\begin{aligned}
\Psi^{\sigma} & =\Gamma T^{-1}(\sigma) \\
& =\left[\begin{array}{llll}
4-9 \sigma^{4} & 12 & 13-10 \sigma^{2} & 6
\end{array}\right] .
\end{aligned}
$$

By performing the coordinate transformation (17), we can obtain the following augmented system

$$
\begin{aligned}
& \dot{\bar{z}}=\bar{f}(\bar{z}, e, \mu) \\
& \dot{\bar{\eta}}=M \bar{\eta}+M N b^{-1} e-N b^{-1} \bar{g}\left(\bar{z}_{1}, \bar{z}_{2}, e, \mu\right) \\
& \dot{e}=\bar{g}\left(\bar{z}_{1}, \bar{z}_{2}, e, \mu\right)+\Psi^{\sigma}(b \bar{\eta}+N e)+b\left(u-\Psi^{\sigma} \eta\right)
\end{aligned}
$$

where $\quad \bar{z}=\left(\bar{z}_{1}, \bar{z}_{2}\right)^{\top}, \quad \bar{f}(\bar{z}, e, \mu)=\left(a_{1} \bar{z}_{1}+a_{2} e, a_{3} \bar{z}_{2}+\left(\bar{z}_{1}+\mathbf{z}_{1}\right)\left(e+v_{1}\right)-\mathbf{z}_{1} v_{1}\right)^{\top}$ and $\bar{g}\left(\bar{z}_{1}, \bar{z}_{2}, e, \mu\right)=a_{5} e+a_{4} \bar{z}_{1}-\left(\bar{z}_{1}+\mathbf{z}_{1}\right)\left(\bar{z}_{2}+\mathbf{z}_{2}\right)+\mathbf{z}_{1} \mathbf{z}_{2}$.

For the $\bar{z}$-subsystem, let $V(\bar{z})=\frac{\ell}{2} \bar{z}_{1}^{2}+\frac{\ell}{4} \bar{z}_{1}^{4}+\frac{1}{2} \bar{z}_{2}^{2}$ for some $\ell>0$. Then for $\mu \in \Sigma \subset \mathbb{V} \times \mathbb{W} \times \mathbb{S}$, there exist some constants $\hbar_{i}>0, i=1, \ldots, 5$, such that, $\frac{\partial V(\bar{z})}{\partial \bar{z}} \bar{f}(\bar{z}, e, \mu) \leq-\hbar_{1} \bar{z}_{1}^{2}-\hbar_{2} \bar{z}_{1}^{4}-\hbar_{3} \bar{z}_{2}^{2}+\hbar_{4} e^{2}+\hbar_{5} e^{4}$, and therefore Assumption 5 is satisfied. By Theorem 1, Problem 1 for system (43) is solved by an output feedback controller in the form of (31) with $\rho(e)=2\left(1+e^{6}+\frac{1}{\left(k_{b}^{2}-e^{2}\right)^{2}}\right)$.

The system is simulated with $\sigma=0.8, \quad \bar{\Omega}=(-9.9,9.8,-8 / 3,28,-1)^{\top}, \quad w=$ $(-0.1,0.2,0,0,0)^{\top}$ and $b=1$. The initial conditions $\left(z_{1}(0), z_{2}(0)\right)=(3,-1)^{\top}, \eta(0)=$ 
$\hat{\Psi}(0)=0, k_{b}(0)=0.73$ and $k(0)=1$. In order to illustrate the performance of the proposed BLF-based control, the representative initial value of the controlled output $y(0)$ is set to be $y(0)=9.72$ such that $e(0)=0.72$ satisfying $e(0)<k_{b}(0)$. It can be seen from Figure 1 that $y(t)$ tracks the desired trajectory $v_{1}(t)$ while satisfying $y(t)<k_{c}(t), \forall t>0$. Figure 2 shows that the tracking error converges to zero without constraint violation.

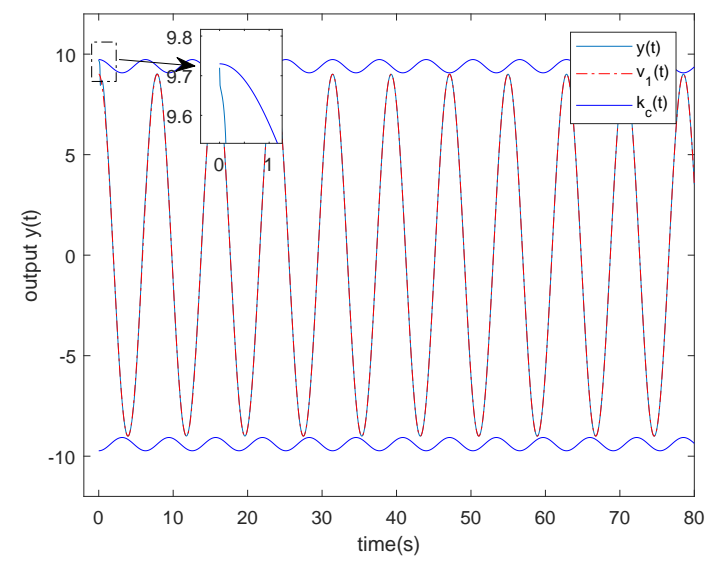

Figure 1. Profile of the controlled output $y(t)$

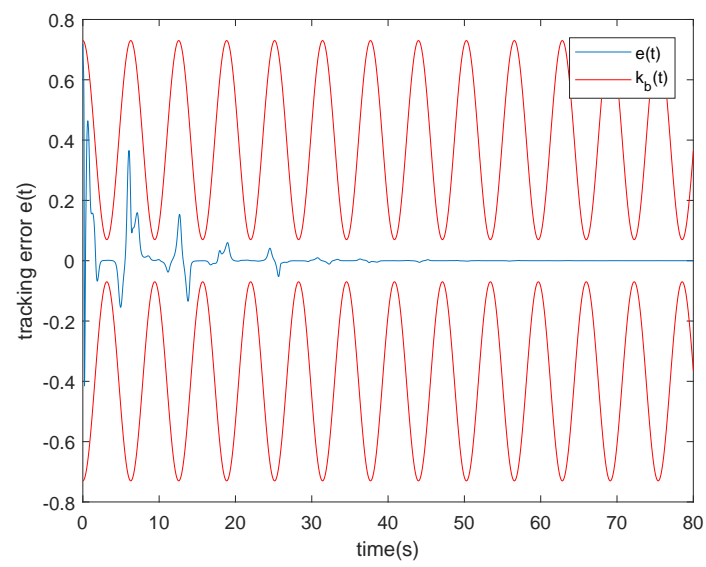

Figure 2. Profile of the tracking error $e(t)$ 
Specially, by Remark 9, the feedback control law ensures the parameter convergence property $\lim _{t \rightarrow \infty}\left(\hat{\Psi}-\Psi^{\sigma}\right)=0$. Figures 3 and 4 show this property for $\sigma=0.8$ with $\Psi^{\sigma}=\left[\begin{array}{llll}0.3136 & 12 & 6.6 & 6\end{array}\right]$. In fact, the estimated frequence $\hat{\sigma}$ can be calculated by the third component of $\hat{\Psi}$, i.e., $\hat{\Psi}_{3}$, as

$$
\hat{\sigma}=\sqrt{1.3-0.1 \hat{\Psi}_{3}}
$$
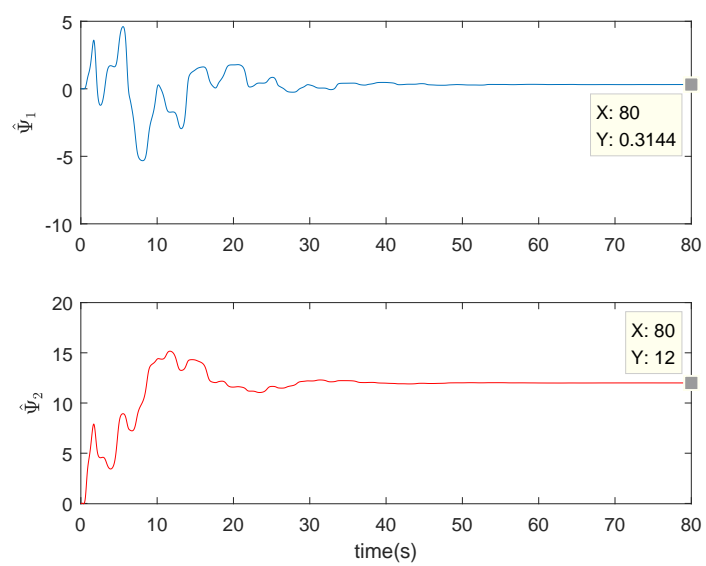

Figure 3. Profile of estimation $\hat{\Psi}$ (first two components)
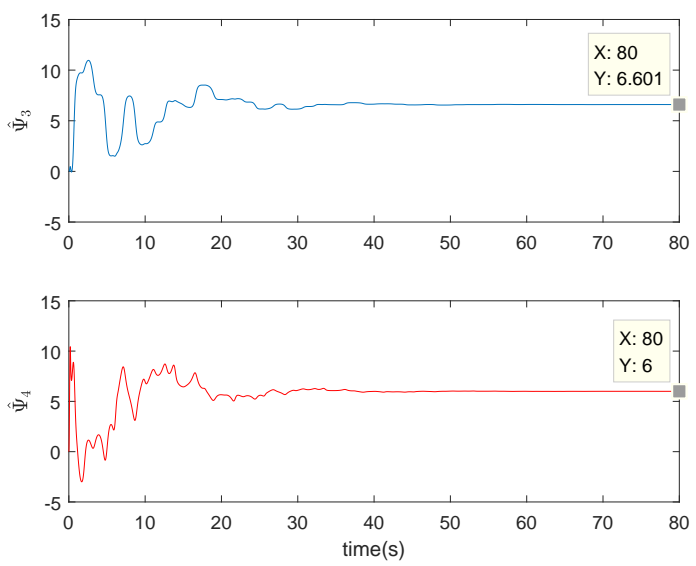

Figure 4. Profile of estimation $\hat{\Psi}$ (last two components) 
Figure 5 shows that the estimated frequency $\hat{\sigma}$ approaches the true frequency. Also, it can be observed in Figure 6 that the dynamic gain $k(t)$ converges to a constant value after some time. In summary, the simulation results show the satisfactory transient performance of the proposed adaptive output regulation design with guaranteed transient constraint for the controlled output.

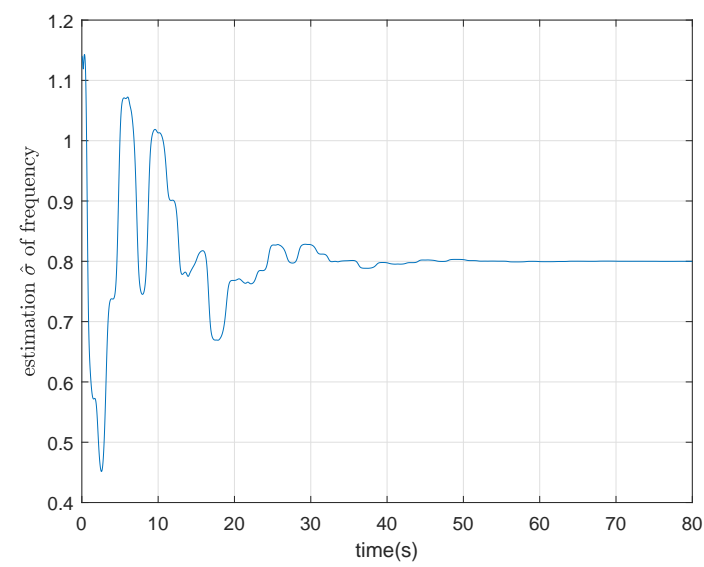

Figure 5. Profile of estimated frequency $\hat{\sigma}$

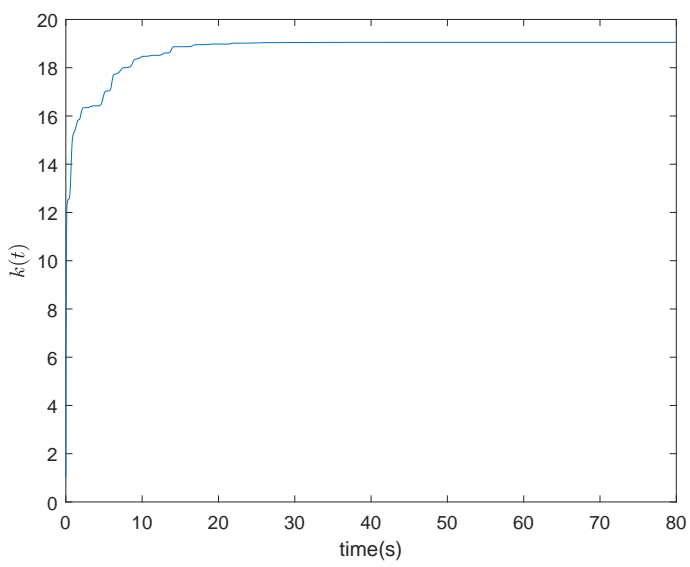

Figure 6. Profile of dynamic gain $k(t)$ 
Under the same initial conditions and the same system parameters, we also compare our output regulation design using the BLF technique with the one using the QLF. Results for the QLF-based design are depicted in Figures 7 and 8. It can be seen from Figure 7 that though the trajectory of $y(t)$ converges to the desired trajectory $v_{1}(t)$, the output constraint is violated. The corresponding error trajectory also deteriorates with constraint violation, as shown in Figure 8. These simulation results demonstrate the effectiveness of the BLF-based design in terms of having transient performance guarantees.

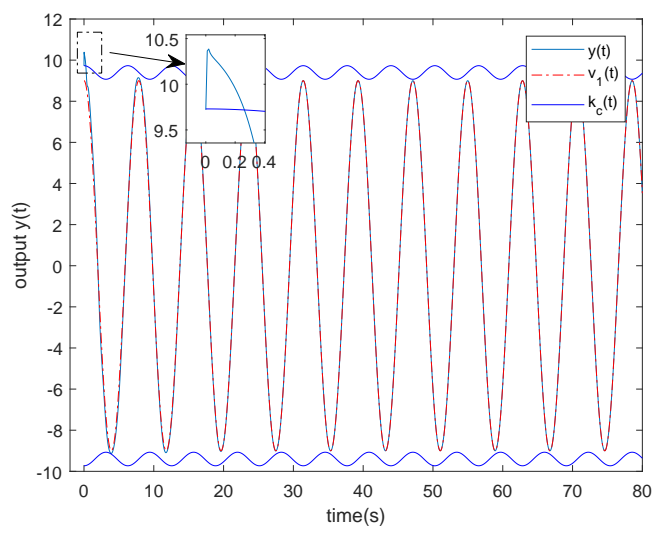

Figure 7. Profile of the output trajectory $y(t)$ with QLF

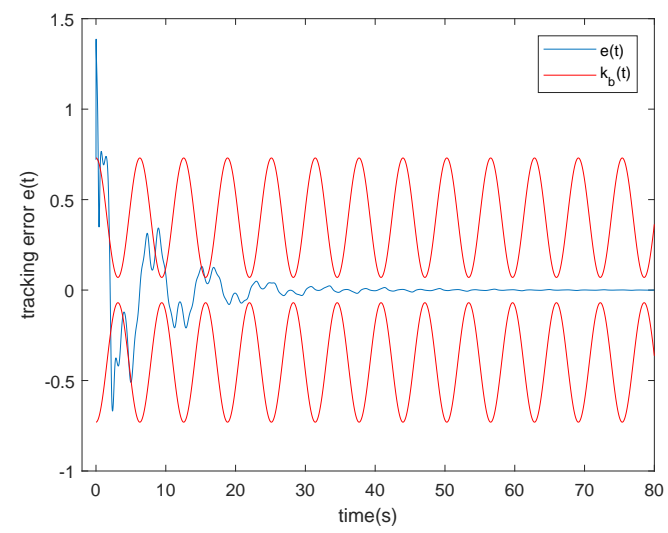

Figure 8. Profile of the tracking error $e(t)$ with QLF 


\section{Conclusion}

In this paper, we have studied the adaptive output regulation problem for a class of nonlinear systems with prescribed transient performance guarantees for the controlled output. Based on the internal model principle, we have transformed this problem into a specific adaptive stabilization problem with output constraints. For the constrained stabilization problem, we have employed the TVBLF to prevent the controlled output from violating the required time-varying constraints. Moreover, the high gain technique is incorporated to cover the unknown uncertain parameters and exogenous signals. Based on the stability analysis of the closed-loop system, we have shown that the trajectory of the closed-loop system is bounded and the controlled output can asymptotically track the desired reference, and, in particular, the time-varying constraints imposed on the output will never be violated. The proposed design is demonstrated to be effective through the simulation results. However, there also exists a tradeoff between the allowed control signal and the convergence performance in the BLF-based design. The future research direction would include extending the design methodology to the event-triggered control of nonlinear multi-agent systems.

\section{Acknowledgements}

The work was supported in part by the National Natural Science Foundation of China under Grant 61573154, and in part by Science \& Technology Planning Project of Guangdong Province under Grant 2015A010106003 and Grant 2017A010101009.

\section{References}

Devasia S, Eleftheriou E and Moheimani SOR (2007) A survey of control issues in nanopositioning. Transactions on Control Systems Technology 15(5): 802-823.

Guo M and Liu L (2018) Output regulation of output feedback systems with unknown exosystem and unknown high-frequency gain sign. Transactions of the Institute of Measurement \& Control 40(1): 171-178. 
He W, Chen Y and Yin Z (2016a) Adaptive neural network control of an uncertain robot with full-state constraints. IEEE Transactions on Cybernetics 46(3): 620-629.

He W, David AO, Yin Z and Sun C (2016b) Neural network control of a robotic manipulator with input deadzone and output constraint. IEEE Transactions on Systems, Man, and Cybernetics: Systems 46(6): 759-770.

He W, Sun C and Ge SS (2015) Top tension control of a flexible marine riser by using integralbarrier lyapunov function. IEEE/ASME Transactions on Mechatronics 20(2): 497-505.

Huang J (2003) On the solvability of the regulator equations for a class of nonlinear systems. IEEE Transactions on Automatic Control 48(5): 880-885.

Huang J (2004) Nonlinear Output Regulation: Theory and Applications. Philadelphia: PA: SIAM.

Huang J and Chen Z (2004) A general framework for tackling the output regulation problem. IEEE Transactions on Automatic Control 49(12): 2203-2218.

Isidori A and Byrnes CI (1990) Output regulation of nonlinear systems. IEEE Transactions on Automatic Control 35(2): 131-140.

Kristic M, Kanellakopoulos I and Kokotovic P (1995) Nonlinear and adaptive control design. New York: Wiley.

Li D, Liu Y, Tong S, Chen CLP and Li D (2019) Neural networks-based adaptive control for nonlinear state constrained systems with input delay. IEEE Transactions on Cybernetics 49(4): $1249-1258$.

Li H, Zhao S, He W and Lu R (2019) Adaptive finite-time tracking control of full state constrained nonlinear systems with dead-zone. Automatica 100: 99 - 107.

Lin X, Lin D, Zhang and Lan W (2017) Semi-global output regulation for discrete-time singular linear systems with input saturation via composite nonlinear feedback control. Transactions of the Institute of Measurement \& Control 39(3): 352-360.

Lin Z, Pachter M and Banda S (1998) Toward improvement of tracking performance nonlinear feedback for linear systems. International Journal of Control 70(1): 1-11.

Liu L (2015) Adaptive cooperative output regulation for a class of nonlinear multi-agent systems. IEEE Transactions on Automatic Control 60(6): 1677-1682. 
Liu L, Chen Z and Huang J (2009) Parameter convergence and minimal internal model with an adaptive output regulation problem. Automatica 45(5): 1306-1311.

Liu T and Jiang ZP (2016) Further results on quantized stabilization of nonlinear cascaded systems with dynamic uncertainties. Science China Information Sciences 59(7): 072202-1-07220212.

Liu W and Huang J (2017) Event-triggered global robust output regulation for a class of nonlinear systems. IEEE Transactions on Automatic Control 62(11): 5923-5930.

Liu Y, Lu S, Tong S, Chen X, Chen CP and Li D (2018) Adaptive control-based barrier lyapunov functions for a class of stochastic nonlinear systems with full state constraints. Automatica 87: $83-93$.

Liu Y and Tong S (2016) Barrier Lyapunov functions-based adaptive control for a class of nonlinear pure-feedback systems with full state constraints. Automatica 64(4): 70-75.

Lu M and Huang J (2015) A class of nonlinear internal models for global robust output regulation problem. International Journal of Robust and Nonlinear Control 25(12): 1831-1843.

Luo S and Song Y (2016) Chaos analysis-based adaptive backstepping control of the microelectromechanical resonators with constrained output and uncertain time delay. IEEE Transactions on Industrial Electronics 63(10): 6217-6225.

Nikiforov VO (1998) Adaptive nonlinear tracking with complete compensation of unknown disturbances. European Journal of Control 4(2): 132-139.

Sontag ED and Teel AR (1995) Changing supply functions in input/state stable systems. IEEE Transaction on Automatic Control 40(8): 1476-1478.

Su Y, Xu L, Wang X and Xu D (2019) Event-based cooperative global practical output regulation of multi-agent systems with nonlinear leader. Automatica 107: 600-604.

Sun W and Huang J (2009) Output regulation for a class of nonlinear systems with nonlinear exosystem and its application. Science in China, Ser. F: Information Sciences 52(11): 21722179.

Sun W, Lan J and Yeow JTW (2015) Constraint adaptive output regulation of output feedback systems with application to electrostatic torsional micromirror. International Journal of Robust \& Nonlinear Control 25(4): 504-520. 
Sun W, Yeow JTW and Sun Z (2012) Robust adaptive control of a one degree of freedom electrostatic microelectromechanical systems model with output-error-constrained tracking. IET Control Theory and Applications 6(1): 111-119.

Tee KP, Ge SS, and Tay EH (2009) Barrier Lyapunov functions for the control of outputconstrained nonlinear systems. Automatica 45(4): 918-927.

Tee KP, Ge SS and Tay FEH (2009) Adaptive control of electrostatic microactuators with bidirectional drive. IEEE Transactions on Control Systems Technology 17(2): 340-352.

Tee KP, Ren B and Ge SS (2011) Control of nonlinear systems with time-varying output constraints. Automatica 47(11): 2511-2516.

Xu D and Huang J (2009) Output regulation design for a class of nonlinear systems with an unknown control direction. Journal of Dynamic Systems, Measurement, and Control 132(1): 014503-1-014503-6.

Xu D and Huang J (2010) Robust adaptive control of a class of nonlinear systems and its applications. IEEE Transactions on Circuits and Systems I: Regular Papers 57(3): 691-702.

Yang C, Jiang Y, Li Z, He W and Su C (2017) Neural control of bimanual robots with guaranteed global stability and motion precision. IEEE Transactions on Industrial Informatics 13(3): $1162-1171$.

Zhang B and Lan W (2013) Improving transient performance for output regulation problem of linear systems with input saturation. International Journal of Robust and Nonlinear Control 23(10): 1087-1098. 